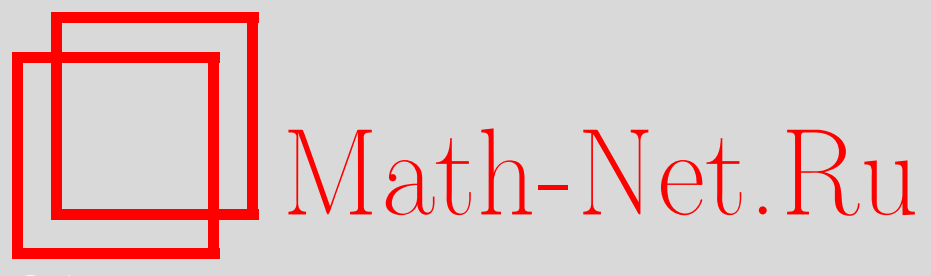

О. Р. Мусин, Обратная теорема об эквивариантных родах, УМH, 2009, том 64, выпуск 4, 179-180

DOI: https://doi.org/10.4213/rm9302

Использование Общероссийского математического портала Math-Net.Ru подразумевает, что вы прочитали и согласны с пользовательским соглашением http://www . mathnet.ru/rus/agreement

Параметры загрузки:

IP: 34.229 .108 .108

26 апреля 2023 г., 13:54:32

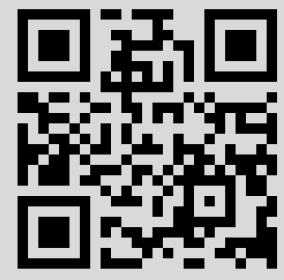




\section{Обратная теорема об эквивариантных родах}

\section{О.Р. Мусин}

Род Хирцебруха, т. е. гомоморфизм $h: \Omega^{*} \rightarrow R$, где $\Omega^{*}=U^{*}(p t)-$ кольцо комплексных кобордизмов и $R=\mathbb{Q}, \mathbb{R}$ или $\mathbb{C}$ (кольцо рациональных, действительных или комплексных чисел), с точки зрения характеристических классов задается рядом $H(t) \in R[[t]]$, так что $h\left(\left[\mathbb{C P}^{n}\right]\right)=\left[H(t)^{n+1}\right]_{n}$. С. П. Новиковым [1] было доказано, что ряд $t / H(t)$ совпадает с рядом $g_{h}^{-1}(t)$, функционально обратным логарифму $g_{h}(t)=\sum_{n} h\left(\left[\mathbb{C P}^{n}\right]\right) t^{n+1} /(n+1)$.

По каждому роду $h: \Omega^{*} \rightarrow R$ и группе $G$ можно построить эквивариантный род $h_{G}: \Omega_{G}^{*} \rightarrow K(B G) \otimes R$ - гомоморфизм из кольца кобордизмов $G$-многообразий в кольцо $K(B G) \otimes R$ (см. [2], [3]). Назовем род $h$ жестким, если для $G=S^{1}$ образ гомоморфизма $h_{G}$ принадлежит подкольцу $R \subset K(B G) \otimes R=R[[t]]$.

Пусть $x, y \in R$ и $x+y$ - положительное целое число. Тогда $T_{x, y}$-род задается как $T_{x, y}\left(\left[\mathbb{C P}^{n}\right]\right)=\left(x^{n+1}-(-y)^{n+1}\right) /(x+y)$. В работе [2] И. М. Кричевером доказана жесткость $T_{x, y}$-рода, из чего было получено новое доказательство формулы АтьиХирцебруха для неподвижных точек $S^{1}$-многообразий. (Еще одно доказательство этой формулы содержится в [4].) Целью настоящей заметки является теорема, обратная к теореме Кричевера.

Теорема. Если род $h: \Omega^{*} \rightarrow R$ жесткий $и g_{h}^{-1}(\ln z)$ является рациональной функuией от $z$, mo $h$ есть $T_{x, y}$-род.

ДокАЗАтЕльство. По условию $F(z):=g_{h}^{-1}(\ln z)=P_{n}(z) / Q_{m}(z)$, где $P_{n}(z)$ и $Q_{m}(z)$ - многочлены степени $n$ и $m$, не имеющие общих делителей. Заметим, что $F(1)=0$. Без ограничения общности можно считать, что $F^{\prime}(1)=1$ и старший коэффициент $P_{n}(z)$ равен 1.

Для всякого $S^{1}$-многообразия $X$ образ $h_{S^{1}}(X) \in R[[z]]$ может быть выражен через инварианты его множества неподвижных точек [2; теорема 1.2]. Для жесткого рода $h$ ряд $h_{S^{1}}(X)$ является константой в $R[[z]]$.

Рассмотрим стандартное действие окружности (вращение) $\tau$ на $\mathbb{S}^{2}=\mathbb{C P}^{1}$ с двумя неподвижными точками. Тогда (см. [2])

$$
h_{S^{1}}\left(\left[\mathbb{C P}^{1}, \tau\right]\right)=\tilde{h} \circ \psi\left(\mathbb{C P}_{1}^{0}\right)+\tilde{h} \circ \psi\left(\mathbb{C P}_{-1}^{0}\right)=1 / F(z)+1 / F(1 / z) \equiv c_{1},
$$

или

$$
Q_{m}(z) P_{n}^{*}(z)+z^{n-m} Q_{m}^{*}(z) P_{n}(z)=c_{1} P_{n}(z) P_{n}^{*}(z) .
$$

Здесь $f^{*}(z)=z^{d} f(1 / z)$, где $f(z)$ - многочлен степени $d$.

Из (1) вытекает, что если $P_{n}(a)=0$, то $P_{n}^{*}(a)=0$ и $P_{n}(1 / a)=0$. Так как $P_{n}(1)=0$, мы получили разложение

$$
P_{n}(z)=(z-1)(z+1)^{k} \prod_{i}\left(z-a_{i}\right)\left(z-1 / a_{i}\right), \quad k \geqslant 0 .
$$

Следовательно, $P_{n}^{*}(z)=-P_{n}(z)$ и (1) влечет

$$
z^{n-m} Q_{m}^{*}(z)=Q_{m}(z)-c_{1} P_{n}(z) .
$$

Рассмотрим теперь действие $\rho(\varphi):\left[z_{0}: z_{1}: z_{2}\right] \rightarrow\left[z_{0}: z_{1}: e^{i \varphi} z_{2}\right]$ на $\mathbb{C P}^{2}$. Заметим, что множество неподвижных точек $\rho$ есть $\mathbb{C P}^{1} \cup \mathbb{C P}^{0}$. Тогда по формуле Кричевера [2] получаем

$$
h_{S^{1}}\left(\left[\mathbb{C P}^{2}, \rho\right]\right)=\tilde{h} \circ \psi\left(\mathbb{C P}_{1}^{1}+\mathbb{C P}_{-1}^{0} \times \mathbb{C P}_{-1}^{0}\right)=\tilde{h}\left(B_{1}(u) / u^{2}\right)+\tilde{h}\left(1 /[u]_{-1}^{2}\right),
$$

Работа выполнена при поддержке грантов NSF: DMS0807640 и NSA: MSPF-08G-201. 
где

$$
\begin{aligned}
\tilde{h}\left(B_{1}(u)\right) & =\frac{\tilde{h}(u)}{(1 / \tilde{h}(u)) g_{h}^{-1}\left(g_{h}(u)+g_{h}(v)\right)}\left(\bmod v^{2}\right)=\tilde{h}_{v}\left(\frac{F(z)}{(1 / F(z)) F\left(z e^{v}\right)}\right)\left(\bmod v^{2}\right) \\
& =\tilde{h}_{v}\left(\frac{F(z)}{1+z\left(F^{\prime}(z) / F(z)\right) v}\right)\left(\bmod v^{2}\right)=h\left(\left[\mathbb{C P}^{1}\right]\right) F(z)-h\left(\left[\mathbb{C P}^{0}\right]\right) z F^{\prime}(z) .
\end{aligned}
$$

Поскольку $h\left(\left[\mathbb{C P}^{0}\right]\right)=h(\mathbf{1})=F^{\prime}(1)=1$, получаем

$$
\begin{gathered}
h_{S^{1}}\left(\left[\mathbb{C P}^{2}, \rho\right]\right)=\frac{h_{1} F(z)-z F^{\prime}(z)}{F^{2}(z)}+\frac{1}{F^{2}(1 / z)} \equiv c_{2}, \quad h_{1}=h\left(\left[\mathbb{C P}^{1}\right]\right), \\
\left(z^{n-m} Q_{m}^{*}\right)^{2}+h_{1} P_{n} Q_{m}+z P_{n} Q_{m}^{\prime}-z P_{n}^{\prime} Q_{m}=c_{2} P_{n}^{2} .
\end{gathered}
$$

Из (3) следует, что многочлен $\left(z^{n-m} Q_{m}^{*}\right)^{2}-z P_{n}^{\prime} Q_{m}$ делится на $P_{n}$. В силу (2) $\left(Q_{m}(z)-c_{1} P_{n}(z)\right)^{2}-z P_{n}^{\prime} Q_{m}$ делится на $P_{n}$, и, стало быть, $P_{n}$ делит $Q_{m}-z P_{n}^{\prime}$. Так как $m \leqslant n($ см. $(2))$, то $Q_{m}-z P_{n}^{\prime}=c P_{n}$, т. е.

$$
Q_{m}(z)=c P_{n}(z)+z P_{n}^{\prime}(z) .
$$

Отсюда следуют равенства $m=n$ и

$$
Q_{m}^{*}(z)=\left(c-c_{1}\right) P_{n}(z)+z P_{n}^{\prime}(z) .
$$

Поскольку старший коэффициент $P_{n}$ равен 1 , то $P_{n}^{*}(0)=1$. Отсюда $P_{n}(0)=$ $-P_{n}^{*}(0)=-1$. Из (4), (5) следуют равенства $Q_{m}(0)=-c, Q_{m}^{*}(0)=c_{1}-c$. Из (3) следует, что

$$
c_{2}=c h_{1}+\left(c_{1}-c\right)^{2} .
$$

Подставим (4)-(6) в (3). После сокращений и деления на $z P_{n}$ получим $a P_{n}^{\prime}=z P_{n}^{\prime \prime}$, где $a=2 c_{1}-h_{1}-2 c-1$. Нетрудно увидеть, что $a=n-1$ и $P_{n}(z)=z^{n}-1$. В силу (4) получаем $Q_{m}(z)=(c+n) z^{n}-c$. Пусть $x=c+n, y=-c$. Тогда $F(z)=\left(z^{n}-1\right) /\left(x z^{n}+y\right), n=x+y$, что соответствует $T_{x, y}$-роду. Теорема доказана.

ЗАмЕчАния. 1. В работе [5] введено понятие жесткости рода $h$ относителъно многообразия $X$, т. е. образ $h_{S^{1}}(X) \in R[[z]]$ является константой. Из доказательства следует, что для обратной теоремы достаточно условия жесткости рода $h$ относительно $\mathbb{C P}^{1}$ и $\mathbb{C P}^{2}$.

2. Недавно удалось избавиться от условия рациональности $g_{h}^{-1}(\ln z)$. Обратная теорема верна и без этого ограничения (работа в печати).

В работе [6] И. М. Кричевер существенно расширил семейство жестких родов. Интересно найти обратную теорему для этих родов.

Автор весьма обязан И. М. Кричеверу за постановку задачи, В. М. Бухштаберу и С. П. Новикову за полезные обсуждения.

\section{Список литературы}

[1] С. П. Новиков, Изв. АН СССР. Сер. матем., 32:6 (1968), 1245-1263. [2] И. М. Кричевер, Изв. АН СССР. Сер. матем., 38:6 (1974), 1289-1304. [3] В. М. Бухштабер, Н. Рэй, УМН, 62:1 (2007), 195-196. [4] О. Р. Мусин, Матем. сб., 116:3 (1981), 359-369. [5] F. Hirzebruch, T. Berger, R. Jung, Manifolds and modular forms, Aspects Math., Vieweg, Braunschweig, 1992. [6] И. М. Кричевер, Матем. заметки, 47:2 (1990), 34-45.

O. P. Мусин (O. R. Musin)

University of Texas at Brownsville

E-mail: oleg.musin@utb.edu
Представлено И. М. Кричевером Принято редколлегией 16.06.2009 